\title{
MANEJO DE AEROSSÓIS DURANTE A PANDEMIA DA COVID-19
}

\author{
Aerosol management during the COVID-19 pandemic
}

\author{
Manejo de aerosol durante la pandemia COVID-19
}

\author{
Aline Coutinho Sento Sé ${ }^{*}$ (1) Raquel Calado da Silva Gonçalves ${ }^{2}$ (D), Perla da Silva Antunes ${ }^{3}$ (D), \\ Nébia Maria Almeida de Figueiredo $0^{4}$ (D), Teresa Tonini ${ }^{5}$ (C)
}

RESUMO: Objetivo: Descrever o processo de prototipação de um dispositivo de filtragem para manejo de aerossóis em procedimentos laparoscópicos durante a pandemia do coronavírus SARS-CoV-2. Método: Estudo descritivo, tipo relato de experiência sobre o protótipo de dispositivo de filtragem para manejo de aerossóis em laparoscopia durante a pandemia de COVID-19 pelo SARS-CoV-2, com base nos processos de imersão, ideação e prototipação do design thinking. Resultados: Os processos de imersão preliminar e profunda permitiram a abordagem do problema. A utilização do mapa mental proporcionou a identificação dos fatores relacionados às suspensões de cirurgias em decorrência do SARS-CoV-2. Pelas medidas do aspirador das salas cirúrgicas, idealizaram-se os recursos materiais necessários: extensor de aspiração, filtro bacteriológico e viral, conector e ponteira. Conclusão: O dispositivo para filtragem do dióxido de carbono do pneumoperitônio obteve boa aceitação da equipe cirúrgica e foi incorporado à rotina do setor durante a realização de cirurgias laparoscópicas. Palavras-chave: Betacoronavírus. Laparoscopia. Pneumoperitônio artificial. Equipamentos e provisões. Centro cirúrgico hospitalar.

ABSTRACT: Objective: To describe the prototyping process of a filtration device for aerosol management in laparoscopic procedures during the SARSCoV-2 coronavirus pandemic. Method: Descriptive study with the report of experience on the prototype filter device for aerosol management in laparoscopy during the SARS-CoV-2 COVID-19 pandemic, based on immersion, ideation and design thinking prototyping processes. Results: Preliminary and deep immersion processes allowed the problem to be addressed. The use of a mental map helped to identify factors related to surgery suspensions due to the SARS-CoV-2. Based on the measurements of the operating room aspirator, the necessary material resources were idealized: suction extender, bacteriological and viral filter, connector and tip. Conclusion: The device for filtering carbon dioxide from the pneumoperitoneum was well accepted by the surgical team and incorporated into the sector's routine for laparoscopic surgeries.

Keywords: Betacoronavirus. Laparoscopy. Pneumoperitoneum, artificial. Equipment and supplies. Surgery department, hospital.

RESUMEN: Objetivo: Describir el proceso de prototipado de un dispositivo de filtración para el manejo de aerosol en procedimientos laparoscópicos durante la pandemia de Coronavirus SARS-CoV-2. Método: Estudio descriptivo, un relato de experiencia sobre el prototipo de dispositivo de filtrado para el manejo de aerosol en laparoscopia durante la pandemia de COVID-19 por SARS-CoV-2, a partir de los procesos de inmersión, ideación y prototipado del Design Thinking. Resultados: Los procesos de inmersión profunda y preliminar permitieron abordar el problema. El uso del Mapa Mental permitió identificar los factores relacionados con la suspensión de cirugías debido al SARS-CoV-2. A partir de las mediciones del aspirador de las salas quirúrgicas, se idearon los recursos materiales necesarios: extensor de aspiración, filtro bacteriológico y viral, conector y férula. Conclusión: El dispositivo para filtrar dióxido de carbono del neumoperitoneo obtuvo buena aceptación por parte del equipo quirúrgico y fue incorporado a la rutina del sector durante las cirugías laparoscópicas.

Palavras clave: Betacoronavirus. Laparoscopía. Neumoperitoneo artificial. Equipos y suministros. Servicio de cirugía en hospital.

'Doutora em Ciências. Enfermeira especialista em Saúde do Trabalhador e Ecologia Humana no Ministério da Saúde - Rio de Janeiro (RJ), Brasil.

2Doutora em ciências. Enfermeira especialista em Centro Cirúrgico, Recuperação Anestésica e Centro de Material e Esterilização no Ministério da Saúde - Rio de Janeiro (RJ), Brasil.

${ }^{3}$ Enfermeira no Ministério da Saúde - Rio de Janeiro (RJ), Brasil.

«Doutora em Enfermagem. Docente da Escola de Enfermagem Alfredo Pinto, Universidade Federal do Estado do Rio de Janeiro - Rio de Janeiro (RJ), Brasil.

${ }_{5}^{5}$ Doutora em Saúde Coletiva. Docente da Escola de Enfermagem Alfredo Pinto, Universidade Federal do Estado do Rio de Janeiro - Rio de Janeiro (RJ), Brasil.

*Autora correspondente: aline2506@hotmail.com

Recebido: 21/08/2020 - Aprovado: 14/05/2021

https://doi.org/10.5327/Z1414-4425202100020009 


\section{INTRODUÇÃo}

Em dezembro de 2019, o médico Li Wenliang alertou sobre os primeiros casos de uma pneumonia de etiologia desconhecida em Wuhan, província de Hubei, China. Grande parte dos infectados trabalhava ou residia próximo ao mercado local de frutos do mar, onde também se comercializavam animais vivos ${ }^{1,2}$.

Um mês após, o Centro Chinês de Controle e Prevenção de Doenças rastreou, pela amostra de esfregaço coletado de um paciente, o coronavírus da síndrome respiratória aguda grave 2 (SARS-CoV-2). Um novo coronavírus com morbidade e mortalidade consideravelmente maiores que outros vírus pertencentes à mesma família, como o coronavírus do SARS-CoV-2 e o coronavírus da síndrome respiratória no Oriente Médio (Mers-CoV) ${ }^{3}$.

Surgiu, assim, a coronavirus disease 2019 (COVID-19), doença causada pelo SARS-CoV-2, caracterizada por sintomas leves, como uma síndrome gripal, e bom prognóstico na maioria dos pacientes, porém com a possibilidade de sintomas graves de infecção respiratória aguda, com desenvolvimento rápido da síndrome do desconforto respiratório agudo, insuficiência respiratória aguda, pneumonia grave, edema pulmonar, falência de múltiplos órgãos, outras complicações graves e óbito ${ }^{2}$.

No Brasil, identificou-se o primeiro caso confirmado de COVID-19 em 26 de fevereiro de 2020. Tratava-se de homem idoso, residente na cidade de São Paulo (SP), que havia retornado recentemente da Itália. Em 17 de março de 2020, registrou-se o primeiro óbito no país: também idoso, do sexo masculino, residente em São Paulo, que apresentava diabetes e hipertensão, porém sem histórico de viagem ao exterior ${ }^{4}$. No mesmo mês, declarou-se oficialmente a pandemia por SARS-CoV-2 e governos de todo o mundo começaram a implementar estratégias para retardar a propagação da infecção².

Considerando o crescente número de casos confirmados no Brasil, que após quase cinco meses da primeira notificação apresentava um total de 3.997 .865 casos, sendo 3.210.405 recuperados e 123.780 óbitos $^{5}$, emergiram recomendações para o reagendamento dos procedimentos cirúrgicos eletivos, sob a justificativa de disponibilizar leitos e equipamentos de ventilação mecânica para pacientes em estado grave e demanda local aumentada ${ }^{6}$.

Durante a pandemia, orientou-se realizar cirurgias de urgência e emergência mediante um plano de ação, em que toda a equipe multiprofissional estivesse orientada em como proceder, de forma eficaz, perante a utilização de equipamentos de proteção individual (EPI), bem como permitir um quantitativo mínimo de pessoas em sala operatória (SO), a fim de evitar a contaminação dos profissionais ${ }^{6}$.
A realização do ato cirúrgico, por meio de videolaparoscopia, tem-se tornado cada vez mais frequente nos serviços de saúde ${ }^{7}$. Trata-se de técnica cirúrgica minimamente invasiva, que visa diminuir o tempo de internação dos pacientes, reduzir a dor pós-operatória, promover maior conforto no pós-operatório e melhor resultado estético, quando comparado à técnica cirúrgica convencional ${ }^{8}$.

Nas cirurgias por laparoscopia, utiliza-se como recurso o dióxido de carbono $\left(\mathrm{CO}_{2}\right)$ para a expansão do abdome. Esse espaço constitui o pneumoperitônio, no qual a insuflação do gás se dá, geralmente, na cicatriz umbilical, com o emprego de agulha de Veress, permitindo que o cirurgião manuseie instrumentos cirúrgicos para realizar o procedimento. Ao fim, esvazia-se a cavidade abdominal, com a eliminação do $\mathrm{CO}_{2}{ }^{9}$.

Considerando o contexto da pandemia por SARS-CoV-2, especialistas recomendam usar sistemas de ultrafiltragem durante a retirada dos gases e evitar o esvaziamento súbito do pneumoperitônio, enfatizando-se que as incisões para passagem dos instrumentais cirúrgicos devem ser realizadas no menor diâmetro possível, diminuindo a possibilidade de vazamento ao redor dos orifícios. Tais preocupações são pertinentes, uma vez que se relacionam ao risco de aerossolização do SARS-CoV-2 no ambiente e risco de contaminação dos profissionais da saúde envolvidos na assistência ${ }^{10}$.

Em cirurgias laparoscópicas, reiterou-se que o procedimento cirúrgico deveria ser realizado pelo cirurgião mais experiente, com especial cuidado nos momentos de introdução e retirada dos trocateres, verificação das válvulas e borrachas de vedação, diminuição do número de sítios de punção e utilização de mecanismos de filtragem durante a insuflação e desinsuflação do pneumoperitônio, permitindo, assim, a menor possibilidade de dispersão de $\mathrm{CO}_{2}$ para o ambiente cirúrgico ${ }^{11} \mathrm{e}$ exposição dos profissionais ao coronavírus.

Com base na orientação de utilização de sistemas de filtragem para evacuação do pneumoperitônio, norteou-se o problema deste estudo. $\mathrm{Na}$ indisponibilidade desse recurso na instituição hospitalar, como assegurar a realização dos procedimentos cirúrgicos por via laparoscópica com segurança, evitando-se a dispersão de aerossóis contaminantes?

\section{OBJETIVO}

Descrever o processo de prototipação de um dispositivo de filtragem para manejo de aerossóis em procedimentos laparoscópicos durante a pandemia da COVID-19 por SARS-CoV-2. 


\section{MÉTODO}

Estudo descritivo, tipo relato de experiência, sobre o processo de prototipação de um dispositivo de filtragem para procedimentos laparoscópicos, com base no design thinking ${ }^{12}$ e na experiência profissional de enfermeiras experts em centro cirúrgico (CC), atuantes na área há mais de 10 anos.

O cenário deste estudo foi o CC de um hospital público, situado no estado do Rio de Janeiro, com sete SO em funcionamento, utilizadas pelas clínicas cirúrgicas de urologia, ginecologia, vascular, proctologia, pediatria e cirurgia geral. O desenvolvimento da pesquisa compreendeu os meses de maio e junho de 2020 , período da primeira onda da contaminação pelo SARS-CoV-2 no Brasil.

$\mathrm{O}$ design thinking, eixo norteador para o delineamento desta pesquisa, define-se como metodologia criativa para o desenvolvimento de produtos inovadores, abordagem focada na identificação de problemas e construção de soluções, constituído de processos de imersão, ideação e prototipação ${ }^{12,13}$.

A imersão, fase na qual se identificam as necessidades de forma exploratória para analisar o contexto de um problema e estabelecer um entendimento inicial, procedeu-se em dois momentos: imersão preliminar e imersão em profundidade ${ }^{12}$.

No que tange à imersão preliminar, realizaram-se discussões de alinhamento estratégico com uma equipe interdisciplinar sobre as adequações necessárias ao retorno das cirurgias eletivas, até então suspensas em decorrência das medidas restritivas ao avanço da contaminação pelo coronavírus, para o processo de reenquadramento.

Ancorando-se no conceito da pesquisa desk, busca vasta de informações sobre a temática ${ }^{12}$, efetivou-se aprofundamento teórico relacionado aos procedimentos cirúrgicos laparoscópicos e evacuação de $\mathrm{CO}_{2}$ do pneumoperitônio com segurança no transoperatório.

A imersão em profundidade iniciou-se com a elaboração de um plano observacional para mapeamento dos contextos a serem considerados para o desenvolvimento do produto e a solução da problemática. Culminou com a observação em campo operatório, com reflexões e rastreios das enfermeiras especialistas em CC, atinentes às etapas que envolviam o fornecimento, a propagação e a eliminação de $\mathrm{CO}_{2}$ durante procedimentos laparoscópicos.

O pouso no campo, com o olhar voltado a cartografar as necessidades dos usuários diretos da tecnologia, cirurgiões e pacientes, e dos usuários indiretos, demais integrantes da equipe interdisciplinar do bloco cirúrgico, permitiu o delineamento das exigências do produto, o processo de uso e a mitigação do problema relacionado à contaminação do ambiente e, porventura, dos trabalhadores, ao produto do $\mathrm{CO}_{2}$ eliminado da cavidade abdominal do paciente com SARS-CoV-2.

Após o levantamento dos dados na fase de imersão, procedeu-se à análise e à síntese, pela construção de mapa mental, com aplicação web gratuita GoConqr ${ }^{14}$, representação visual por meio de palavras, imagens e cores para gestão de informações e conhecimentos como ferramenta para a compreensão e a solução de problemas ${ }^{15}$.

Posteriormente, instituiu-se o processo de ideação com base na imersão e no mapa mental, ancorando-se nas orientações ${ }^{10}$ para o retorno de cirurgias eletivas durante a pandemia do SARS-CoV-2, revisão do processo assistencial cirúrgico-laparoscópico, avaliação da viabilidade, segurança e usabilidade da tecnologia e justificativa para sua implementação.

A prototipação do dispositivo, transformação da ideia abstrata para o campo físico, de forma que representasse a realidade e permitisse validações ${ }^{12}$, realizou-se no próprio cenário do estudo. Após duas fases de ajustes, elegeu-se o protótipo final para filtragem de $\mathrm{CO}_{2}$ do pneumoperitônio. O dispositivo foi testado e avaliado por cirurgiões e profissionais de enfermagem, que receberam treinamento prévio sobre o objetivo do insumo e a técnica de manuseio e instalação.

\section{RESULTADOS}

Os processos de imersões preliminar e profunda permitiram a abordagem do problema-alvo deste estudo, a necessidade de um dispositivo para realizar filtragem de $\mathrm{CO}_{2}$ do pneumoperitônio de pacientes submetidos a cirurgias laparoscópicas durante a pandemia do SARS-CoV-2, segundo orientações vigentes.

Baseando-se em literaturas específicas à operacionalidade de atividades cirúrgicas, com base na pesquisa desk, discussões interdisciplinares, observação do ambiente cirúrgico, descrição da assistência operatória, infraestrutura, saúde do trabalhador e segurança do paciente, iniciou-se o processo de análise para construir soluções.

A utilização do mapa mental proporcionou a identificação dos fatores relacionados às suspensões de cirurgias em decorrência do SARS-CoV-2 condizentes com pacientes, profissionais de saúde e infraestrutura, síntese, planejamento e estruturação do pensamento, exploração de ideias e manutenção do foco no problema sobre a necessidade de um sistema de filtragem de $\mathrm{CO}_{2}$, conforme disposto na Figura 1.

Após rastrear as primordialidades e cartografar os espaços do cuidar, confeccionou-se relatório de ideação com a 
justificativa para implementação e os insumos disponíveis na instituição para prototipar o dispositivo, objeto deste estudo.

Com base nas medidas do aspirador das SO, idealizaram-se os recursos materiais: extensor de aspiração e filtro bacteriológico e viral (Figura 2).

Em virtude da incompatibilidade dos diâmetros, utilizou-se um conector e uma ponteira como adaptador (Figura 3).

A Figura 4 demonstra a idealização do protótipo para filtragem de $\mathrm{CO}_{2}$ do pneumoperitônio e conexão com o trocater.

A Figura 5 apresenta o dispositivo para filtragem $\mathrm{de}_{2} \mathrm{CO}_{2}$ acoplado em um aspirador portátil e em um aspirador de parede.

O dispositivo foi apresentado às equipes médicas e de enfermagem do bloco cirúrgico, assim como os elementos de sua composição, no intuito de disponibilizar informação para implementar e disseminar o conceito.

\section{DISCUSSÃO}

A metodologia aplicada por meio dos processos de imersão, ideação e prototipação do design thinking, associada à elaboração do mapa mental, permitiu o alcance do objetivo deste estudo.

Recorreu-se à criatividade como medida resolutiva, considerando os impactos negativos à saúde física e emocional dos pacientes decorrentes da espera aumentada para realizar o procedimento cirúrgico, da necessidade de proteção dos profissionais e do ambiente de trabalho, da minimização do risco de contaminações e da dificuldade de aquisição de insumos/equipamentos no serviço público de saúde.

O protótipo desenvolvido foi incorporado à rotina do setor durante a realização de cirurgias videolaparoscópicas, com a utilização de $\mathrm{CO}_{2}$. Ratifica-se o emprego de filtros em

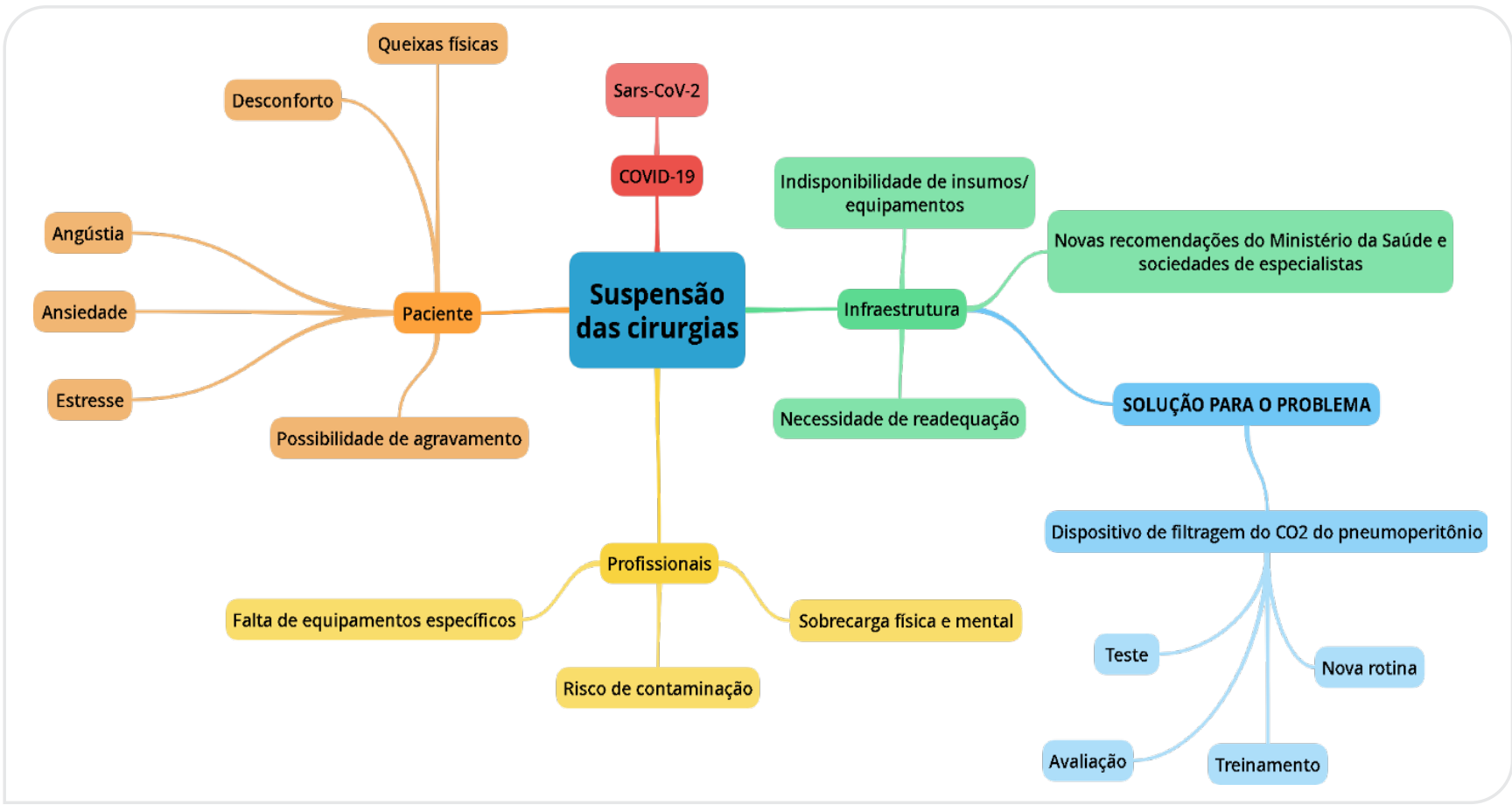

Figura 1. Mapa mental sobre os aspectos relacionados à suspensão das cirurgias.
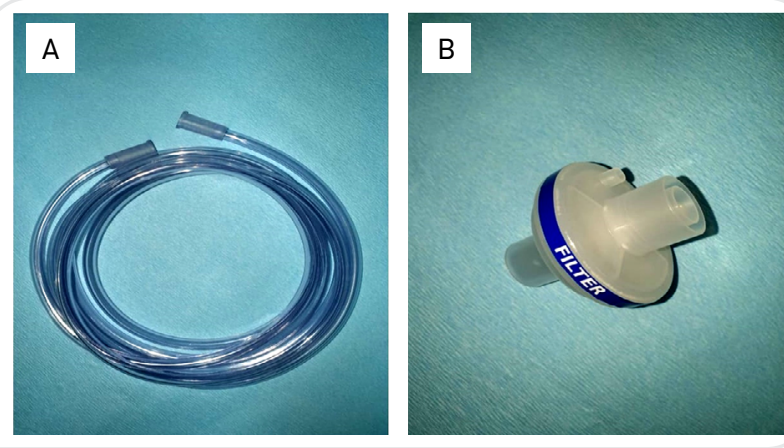

Figura 2. (A) Extensor de aspiração e (B) filtro bacteriológico e viral.
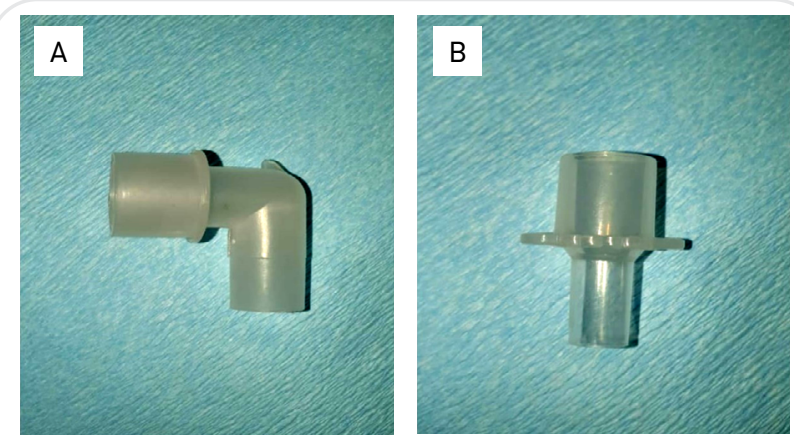

Figura 3. Conector e ponteira. 
cirurgias videolaparoscópicas, salientando que adaptações e associações se fazem necessárias à prevenção e à proteção do risco de infecção dos profissionais da saúde que exercem atividades no enfrentamento do SARS-CoV- $2^{16}$.

Recomenda-se precaução no manejo do pneumoperitônio durante os procedimentos laparoscópicos, durante a insuflação e desinsuflação, por dispersão de aerossóis em SO. Dado o risco potencial de contaminação, cita-se a associação de EPI a dispositivos de barreiras de filtragem do pneumoperitônio como prevenção ao SARS-CoV-2. Sugere-se a utilização de algum dispositivo de filtragem, acoplado em extensor, mantendo distanciamento de, no mínimo, dois metros da equipe operatória, como medida de segurança ${ }^{11,17}$.

Assim como neste estudo, outra pesquisa relatou prototipação de dispositivo com materiais de fácil aquisição no ambiente hospitalar, em virtude da indisponibilidade de dispositivos de filtragem já

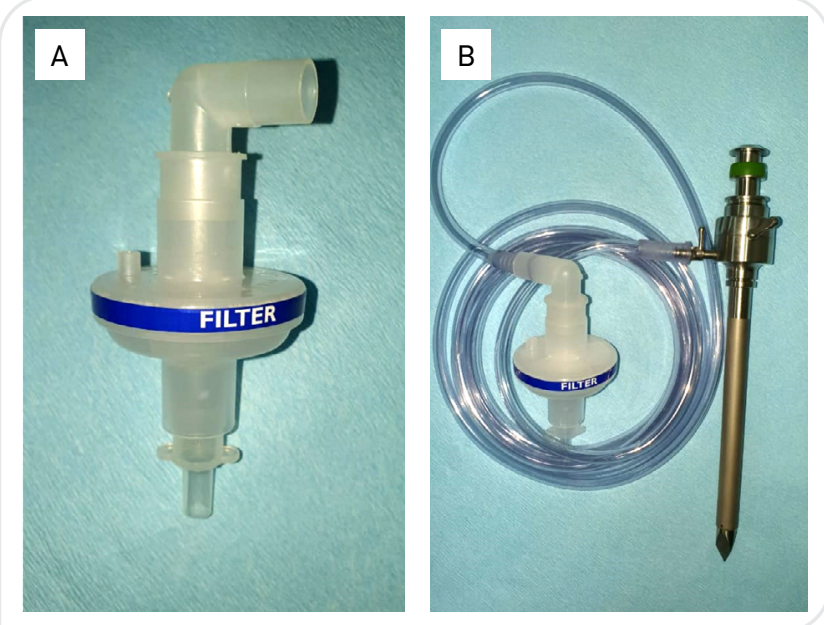

Figura 4. Protótipo para filtragem de $\mathrm{CO}_{2}$ do pneumoperitônio e conexão com o trocater.

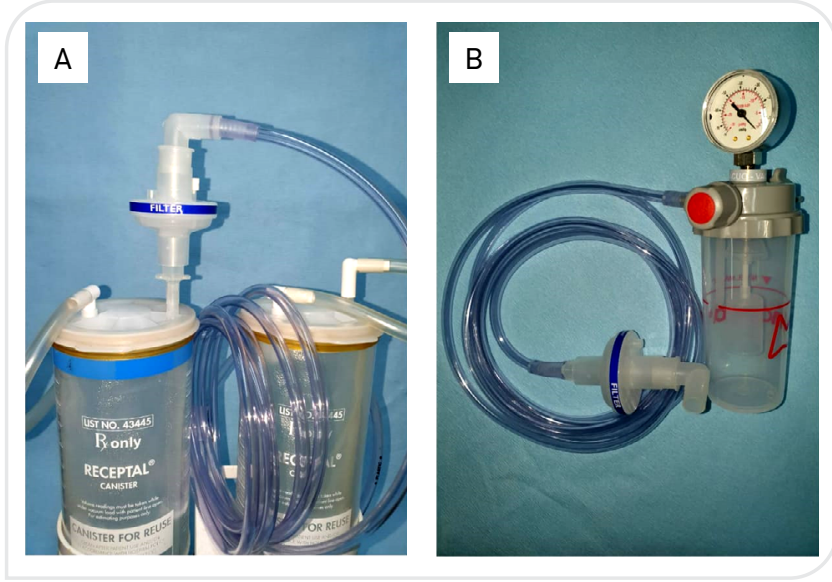

Figura 5. Dispositivo para filtragem de $\mathrm{CO}_{2}$ acoplado em aspirador portátil e em aspirador de parede. comercializados, seja por alta demanda, seja por menor potencial financeiro das instituições de saúde ou órgãos governamentais ${ }^{18}$.

Os procedimentos cirúrgicos iniciais com a utilização do protótipo para filtragem de $\mathrm{CO}_{2}$ do pneumoperitôneo foram acompanhados por uma das autoras, que se responsabilizou pela montagem com a equipe de enfermagem escalada nas $\mathrm{SO}$ e pelas orientações aos instrumentadores cirúrgicos e cirurgiões na fase pré-operatória. Posteriormente, após capacitação dos enfermeiros, circulantes e instrumentadores cirúrgicos do cenário de estudo, incorporou-se o protótipo à rotina do setor durante as cirurgias laparoscópicas. Ressalta-se que não foram identificados vazamentos, dificuldade para o manuseio e desconexões durante os atos cirúrgicos.

Obteve-se boa aceitação da equipe de saúde envolvida no processo cirúrgico. Acredita-se que tal fato esteja relacionado à facilidade da sua composição com materiais já conhecidos pelos profissionais. Por se tratar de prototipação de dispositivo sem financiamento e de caráter emergencial para a manutenção do serviço cirúrgico, assistência aos pacientes e segurança dos trabalhadores, utilizaram-se insumos disponíveis na grade de material hospitalar, com raciocínio criativo ${ }^{13} \mathrm{e}$ expertise dos autores.

No caso dos procedimentos cirúrgicos, aconselha-se avaliação criteriosa quanto à possibilidade de tratamento conservador ${ }^{16} \mathrm{e} / \mathrm{ou}$ adiamento de cirurgias. ${ }^{17}$ Porém, em outros, a abordagem cirúrgica torna-se imprescindível, impactando a rotina de funcionamento dos CC, com área exclusiva para pacientes com suspeita ou certeza diagnóstica do SARS-CoV-2 $2^{17,18}$.

A pandemia decorrente do SARS-CoV-2 tornou obrigatória a implementação de novos protocolos de atendimento, com base em evidências científicas e desenvolvimento de habilidades profissionais, que incluem remodelamentos e substituições de insumos para manutenção de cuidados de saúde essenciais, como os procedimentos cirúrgicos laparoscópicos. Enfatiza-se a implementação de diretrizes institucionais e adequações de práticas cirúrgicas à assistência ao paciente com suspeita ou confirmação de infecção por SARS-CoV-2, a fim de minimizar a exposição dos profissionais de saúde e outros pacientes ao vírus ${ }^{17,19}$.

O estudo apresentou como limitação a validação clínica, última etapa para a ampliação do conhecimento e confiabilidade do dispositivo de filtragem de $\mathrm{CO}_{2}$ do pneumoperitônio como recurso eficaz à prevenção de dispersão de aerossóis no meio ambiente.

\section{CONCLUSÃO}

Conclui-se que os processos do design thinking e a integração dos saberes das enfermeiras experts em CC permitiram o desenvolvimento de um protótipo para o manejo de aerossóis 
em procedimentos videolaparoscópicos, atendendo às recomendações técnicas sobre barreiras de filtragem do pneumoperitônio como prevenção ao SARS-CoV-2.

Trata-se de dispositivo de fácil montagem, com curva de aprendizagem curta, constituído de insumos disponíveis na grade hospitalar, para conferir maior segurança aos profissionais de saúde, bem como aos pacientes.

O protótipo inseriu-se à rotina do cenário do estudo, com boa aceitação da equipe operatória, sendo utilizado durante os procedimentos videolaparoscópicos de pacientes com suspeita ou confirmação de infecção por SARS-CoV-2, durante a primeira onda da pandemia no Brasil.

Reitera-se que a prototipação se deu pela indisponibilidade de recurso à filtragem do $\mathrm{CO}_{2}$ do pneumoperitônio e manutenção do atendimento cirúrgico na instituição. Porém se faz necessário aprofundamento científico, no que tange à validação clínica para avaliar a confiabilidade do dispositivo e a continuidade do estudo.

\section{REFERÊNCIAS}

1. Lauande R, Paula JS. Coronavirus and the eye. Arq Bras Oftalmol. 2020;83(3):5-6. https://doi.org/10.5935/0004-2749.20200057

2. Chen N, Zhou M, Dong X, Qu J, Gong F, Han Y, et al. Epidemiological and clinical characteristics of 99 cases of 2019 novel coronavirus pneumonia in Wuhan, China: a descriptive study. Lancet. 2020;395(10223):50713. https://doi.org/10.1016/S0140-6736(20)30211-7

3. Machhi J, Herskovitz J, Senan AM, Dutta D, Nath B, Oleynikov MD, et al. The natural history, pathobiology, and clinical manifestations of SARS-CoV-2 infections. J Neuroimmune Pharmacol. 2020;15:35986. https://doi.org/10.1007/s11481-020-09944-5

4. Oliveira WK, Duarte E, França GVA, Garcia LP. How Brazil can hold back COVID-19. Epidemiol Serv Saúde. 2020;29(2):e2020044. https:// doi.org/10.5123/s1679-49742020000200023

5. Brasil. Ministério da Saúde. Painel coronavírus [Internet]. Brasil; Ministério daSaúde [acesso em 2 set. 2020]. Disponível em: https://covid.saude.gov.br

6. Correia MITD, Ramos RF, Von Bahten LC. The surgeons and the COVID-19 pandemic. Rev Col Bras Cir. 2020;47:e20202536. https:// doi.org/10.1590/0100-6991e-20202536

7. Berger T, Silva RV, Marui AS, Cicarelli DD. Embolia gasosa por dióxido de carbono durante cirurgia laparoscópica: relato de caso. Rev Bras Anestesiol. 2005;55(1):87-9. https://doi.org/10.1590/S0034-70942005000100010

8. Tuna AT, Akkoyun I, Darcin S, Palabiyik O. Efeitos da insuflação de dióxido de carbono sobre a oxigenação cerebral regional durante cirurgia laparoscópica em crianças: um estudo prospectivo. Rev Bras Anestesiol. 2016;66(3):249-53. https://doi.org/10.1016/j.bjane.2014.10.004

9. CamposFGCM, RollS. Complicações doacessoabdominaledopneumoperitônio em cirurgia laparoscópica: causas, prevenção e tratamento. Rev Bras VideoCir [Internet]. 2003 [acessado em 5 jul. 2020]; 1(1):21-8. Disponível em: https:// www.sobracil.org.br/revista/rv010101/rbvc010101_021.pdf

10. Brasil. Agência Nacional de Vigilância Sanitária. Gerência de Vigilância e Monitoramento em Serviços de Saúde. Nota técnica GVIMS/GGTES/ANVISA n०06/2020. Orientações para a prevenção e o controle das infecções pelo novo coronavírus (SARS-CoV-2) em procedimentos cirúrgicos. Complementarà Nota técnica GVIMS/GGTES/ANVISAn ${ }^{\circ}$ 04/2020. Publicada em 29 de abrilde 2020. Revisão 1: 29 de maio de 2020 [Internet]. Brasil: Agência Nacional de Vigilância Sanitária; 2020 [acessado em 23 jul. 2020]. Disponível em: http:// portal.anvisa.gov.br/documents/33852/271858/Nota+t\%C3\%A9cnica+062020+GVIMS-GGTES-ANVISA/40edaf7d-8f4f-48c9-b876-bee0090d97ae
11. Ramos RF, Lima DL, Benevenuto DS. Recommendations of the Brazilian College of Surgeons for laparoscopic surgery during the COVID-19 pandemic. Rev Col Bras Cir. 2020;47:e20202570. https:// doi.org/10.1590/0100-6991e-20202570

12. Vianna M, Vianna Y, Adler IK, Lucena B, Russo B. Design Thinking: inovação em negócios. Rio de Janeiro: MJV Press; 2012. 164 p.

13. Hauber B, Schreiber D, Pinheiro CMP. Combinando o Design Thinking e a criatividade no processo de inovação aberta. Gestão Planej. 2019;20:73-89. https://doi.org/10.21714/2178-8030gep.v.20.4823

14. GoConqr [Internet]. [acessado em 15 jul. 2020]. Disponivel em: https:// www.goconqr.com/pt-BR

15. Pimentel CF, Pessi DD. Panorama dos artigos sobre mapas mentais publicados na Scientific Periodicals Eletronic Library - SPELL e na Scientific Library Online -SCIELO. Repad [Internet]. 2019 [acessado em 15 jul. 2020];3(2):69-81. Disponível em: https://periodicoscientificos. ufmt.br/ojs/index.php/repad/article/view/8553/6120. https://doi. org/10.30781/repad.v3i2.8553

16. Lima DS, Leite Filho JAD, Gurgel MVSA, Aguiar Neto AF, Costa EFM, Maia Filho FXF, et al. Recomendações para cirurgia de emergência durante a pandemia do COVID-19. J Health Biol Sci. 2020;8(1):1-3. https://doi.org/10.12662/2317-3076jhbs.v8i1.3176.p1-3.2020

17. Mano GBC, Mano GBC, Oliveira GD, Mano RBC, Sardenberg RAS. Emergências cirúrgicas durante a pandemia de COVID-19. ULAKES J Med [Internet]. 2020 [acessado em 4 maio 2021];1:168-79. Disponível em: http://revistas.unilago.edu.br/index.php/ulakes/ article/view/266/257

18. Morrell ALG, Tustumi F, Morrel Júnior AC, Morrel AG, Ribeiro DMFR, Corsi PR, et al. Manejo intraoperatório em cirurgia laparoscópica ou robótica para minimizar a dispersão de aerossóis: adaptações ao contexto da pandemia por COVID-19. Rev Col Bras Cir. 2020;47:e20202558. https://doi.org/10.1590/0100-6991e-20202558

19.Zucco L, Levy N, Ketchandji D, Aziz M, Ramachandran SK. Atualização sobre as considerações perioperatórias para a Síndrome Respiratória Aguda Grave Coronavírus-2 (Sars-Cov-2) do COVID-19. Boletim da APSF [Internet]. 2020 [acessado em $1^{\circ}$ maio 2021];3(2):35-9. Disponível em: https://www.apsf.org/pt-br/ article/atualizacao-sobre-as-consideracoes-perioperatorias-paraa-sindrome-respiratoria-aguda-grave-coronavirus-2-sars-cov2-do-covid-19/ 